\title{
MONOTONE ITERATIONS FOR NONLINEAR OBSTACLE PROBLEM
}

\author{
PHILIP KORMAN, ANTHONY W. LEUNG, AND SRDJAN STOJANOVIC ${ }^{1}$
}

(Received 30 March 1988; revised 24 February 1989)

\begin{abstract}
The existence, uniqueness and regularity of solutions are proved for the obstacle problem with semilinear elliptic partial differential equations of second order. Computationally effective algorithms are provided and application made to steady state problem for the logistic population model with diffusion and an obstacle to growth.
\end{abstract}

\section{Introduction}

We consider the obstacle problem for semilinear elliptic equations of second order, and prove existence, uniqueness and regularity of solutions and provide computationally effective algorithms. We apply our results to the steady state problem for the logistic population model with diffusion and an obstacle to growth. (With this application in mind we require that the solution lies below the obstacle.)

Our proof of existence in the Theorem 1 is based on constructing a monotone sequence of iterations, converging to a solution. On each step one solves the obstacle problem for a linear elliptic equation. For the initial iterate one may take either the obstacle itself, or a subsolution, whose existence we assume. Our solution then lies above the subsolution, which, in particular, allows one to conclude existence of a nontrivial solution in the presence of a trivial one.

Compared with the standard monotone scheme for elliptic equations (see e.g. [1]), the situation is more involved here, since each iterate is not a classical

\footnotetext{
'Department of Mathematical Sciences, University of Cincinnati, Cincinnati, Ohio 45221-0025, U.S.A.

(C) Copyright Australian Mathematical Society 1990, Serial-fee code 0334-2700/90
} 
solution (it is only in $W^{2, p}$ and $W_{l o c}^{2, \infty}$ ), which complicates the passage to the limit. To pass to the limit, we derive $W^{2, p}$ bounds for the approximating (or penalized) problem, which are independent of the approximation parameters $\varepsilon$ and $N$, and the iteration index $n$. We then pass to the limit along a diagonal subsequence, as $\varepsilon \rightarrow 0, N, n \rightarrow \infty$.

Compared with the variational inequalities techniques (see e.g., [3], [2]), we do not assume any special structure upon the nonlinear perturbation $f(x, u)$, such as the sign condition $f(x, u) u>0$. (Although we do assume Lipschitz continuity of $f$ in $u$.)

In the Theorem 2, we prove uniqueness of the positive solution for a general class of equations, including the logistic model. In the Theorem 3 we prove regularity of free boundary, based on the results of L. A. Caffarelli [4], D. Kinderlehrer and L. Nirenberg [7], see also A. Friedman [5].

In Section 4 we develop a Newton-like method with monotone convergence for the obstacle problem (Theorem 5). The advantages of the method are its quadratic convergence and a large domain of attraction for the solution (initial guess does not have to be close to the solution). Also (as in the Theorem 1) we have an automatic error bound on each step. In the Theorem 4 we present an analogous method for semilinear elliptic problems. A similar approach was used previously by R. Bellman, R. Kalaba and their coworkers, and developed by D. Cohen and H. Keller, see e.g., H. Keller [9] and the references therein. However, it is required in [9] that $L-f_{u}(x, 0)$ is a positive (or inverse-positive) operator, a rather restrictive condition, which is not assumed in the Theorem 4.

We shall use the standard spaces $C^{2+\alpha}, W^{m, p}$ with $\|\cdot\|_{m, p}$ denoting the norm in $W^{m, p}$ (see e.g., [5]). We shall write $c$ for all irrelevant positive constants. By $\lambda_{1}$ we denote the principal eigenvalue of $-\Delta$ on $\Omega$ with zero Dirichlet data, and by $\varphi_{1}>0$ the corresponding eigenfunction with $\max _{\Omega} \varphi_{1}=1$, i.e., $\Delta \varphi_{1}+\lambda_{1} \varphi_{1}=0$ in $\Omega, \varphi_{1}=0$ on $\partial \Omega$.

2. Existence, uniqueness and the regularity results

Let $L$ denote the operator

$$
L u \equiv-\sum_{i, j=1}^{n} a_{i j}(x) \frac{\partial^{2} u}{\partial x_{i} \partial x_{j}}+\sum_{i=1}^{n} b_{i}(x) \frac{\partial u}{\partial x_{i}}+c(x) u
$$


$\Omega$ a bounded domain in $R^{n}$. We consider the obstacle problem

$$
\left.\begin{array}{rl}
L u & \leq f(x, u) \\
u & \leq q(x) \\
(L u-f(x, u))(u-q) & =0 \\
u=g(x) & \text { on } \partial \Omega
\end{array}\right\} \text { a.e. in } \Omega
$$

Assume that $L$ is uniformly elliptic operator with coefficients in $C^{\alpha}(\bar{\Omega})$, $q(x) \in C^{2}(\Omega), g(x) \in C^{2+\alpha}(\Omega), g(x)<q(x)$ on $\partial \Omega, \partial \Omega \in C^{2+\alpha}$.

THEOREM 1. In addition to the conditions above, assume that there exists a function $\varphi \in C^{2}(\bar{\Omega})$ (a subsolution), such that

$$
\begin{gathered}
L \varphi \leq f(x, \varphi), \varphi(x) \leq q(x) \quad \text { in } \Omega, \\
\varphi(x) \leq g(x) \text { on } \partial \Omega,
\end{gathered}
$$

and that $f(x, u)$ is $C^{\alpha}$ in $x$ and uniformly Lipschitz in $u$, for $x \in \bar{\Omega}$ and $\varphi(x) \leq$ $u \leq q(x)$. Then the problem (2.1) has a solution $u(x) \in W^{2, p}(\Omega) \cap W_{\text {loc }}^{2, \infty}(\Omega)$ for any $p$. Moreover, $u \geq \varphi(x)$.

Proof. Without loss of generality we may assume that the function $f$ is increasing in $u$ and $c(x) \geq 0$ for all $x \in \Omega$. (Substitute $f$ by $f+M u$, and $L$ by $L+M u$, where $M$ is large.) Also, we may assume that the set $\{x \in \Omega \mid L q<f(x, q)\}$ is nonempty, for otherwise $q(x)$ is a supersolution, and then there exists a solution $u(x)$ for the problem $L u=f(x, u)$ in $\Omega$, $u=g(x)$ on $\partial \Omega$, which is also a solution of (2.1), see e.g., [2].

Let $u^{0}=q(x)$, and define a sequence of functions $\left\{u^{n}(x)\right\}$ by solving the following variational inequality $(n=0,1, \ldots)$

$$
\begin{gathered}
L u^{n+1} \leq f\left(x, u^{n}\right) \\
u^{n+1} \leq q(x) \\
\left(L u^{n+1}-f\left(x, u^{n}\right)\right)\left(u^{n+1}-q\right)=0 \\
u^{n+1}=g(x) \text { on } \partial \Omega .
\end{gathered}
$$

Since $f\left(x, u^{n}\right) \in C^{\alpha}(\bar{\Omega})$, the problem (2.2) has a unique solution $u^{n+1}(x) \in$ $W^{2, p}(\Omega)$ for any $p([6$, p. 26$])$.

We show next that the sequence $\left\{u^{n}(x)\right\}$ is monotone decreasing, i.e.,

$$
u^{0} \geq u^{1} \geq u^{2} \geq \cdots \text {. }
$$

The proof is by induction. Let $k$ be the first index where (2.3) is violated, i.e., the (open) set $G=\left\{x \in \Omega \mid u_{k-1}(x)<u_{k}(x)\right\}$ is non-empty. Then $u_{k-1}<q(x)$ in $G$, and hence by (2.2),

$$
L u^{k-1}=f\left(x, u^{k-2}\right) \text { in } G,
$$


while $L u^{k} \leq f\left(x, u^{k-1}\right)$. By the inductive assumption,

$L\left(u^{k-1}-u^{k}\right) \geq f\left(x, u^{k-2}\right)-f\left(x, u^{k-1}\right) \geq 0 \quad$ in $G, \quad u^{k-1}-u^{k}=0 \quad$ on $\partial G$,

which leads to contradiction by the maximum principle. Similarly, we see that $u^{n}(x) \geq \varphi(x)$ for any $n$.

The monotone sequence $\left\{u^{n}(x)\right\}$ will converge pointwise to a function $u(x) \geq \varphi(x)$. We proceed to show that $u(x)$ solves (2.1). For this we prove the estimate

$$
\left\|u^{n}\right\|_{2, p} \leq c \text { for any } p \text {, independently of } n \text {. }
$$

It will imply that $u^{n} \rightarrow u$ weakly in $W^{2, p}(\Omega)$ for any $p$, and hence uniformly in $\bar{\Omega}$.

Recall that $u^{n+1}$ can be obtained as a limit when $\varepsilon \rightarrow 0$ (along some sequence $\varepsilon=\varepsilon_{m}$ ) of the solution $u_{\varepsilon}^{n}$ of the penalized problem ([6]),

$$
\begin{aligned}
L u_{\varepsilon}^{n+1}+\beta_{\varepsilon}\left(u_{\varepsilon}^{n+1}-q\right) & =f\left(x, u^{n}\right) \quad \text { in } \Omega \\
u_{\varepsilon}^{n+1} & =g(x) \text { on } \partial \Omega .
\end{aligned}
$$

Here $\beta_{\varepsilon}(t)(0<\varepsilon<1)$ is a family of $C^{\infty}$ in $t$ functions, satisfying the properties

$$
\begin{gathered}
\beta_{\varepsilon}^{\prime}(t)>0 \text { for }-\infty<t<\infty, 0<\varepsilon<1, \\
\beta_{\varepsilon}(t) \rightarrow 0 \text { for } t<0, \varepsilon \rightarrow 0, \\
\beta_{\varepsilon}(t) \rightarrow \infty \quad \text { if } t>0, \varepsilon \rightarrow 0, \\
\beta_{\varepsilon}(t) \geq-c, \beta_{\varepsilon}(0) \leq c \quad(c \text { is independent of } \varepsilon) .
\end{gathered}
$$

Recall that to solve (2.5) one introduces $\beta_{\varepsilon, N}(t)=\max \left\{\min \left(\beta_{\varepsilon}(t), N\right),-N\right\}$, and considers the problem

$$
\begin{aligned}
L u_{\varepsilon, N}^{n+1}+\beta_{\varepsilon, N}\left(u_{\varepsilon, N}^{n+1}-q\right) & =f\left(x, u^{n}\right) \quad \text { in } \Omega \\
u_{\varepsilon, N}^{n+1} & =g(x) \quad \text { on } \partial \Omega .
\end{aligned}
$$

By the Schauder's fixed point theorem and elliptic regularity, problem (2.6) has a solution in $C^{2+\alpha}(\Omega)$.

We now proceed with the inductive proof of (2.5) assuming it to hold up to an index $n$. We need a bound on the function $\xi(x) \equiv \beta_{\varepsilon, N}\left(u_{\varepsilon, N}^{n+1}-q\right)$. By the definition of $\beta_{\varepsilon}, \xi(x) \geq-c$. Let now $x^{0}$ be the point of maximum $\xi(x)$. We may assume that $\xi\left(x^{0}\right)>\beta_{\varepsilon}(0)$ for $0<\varepsilon<1$. Notice that $x^{0} \notin \partial \Omega$, for otherwise

$$
\xi\left(x^{0}\right)=\beta_{\varepsilon, N}(g-q) \leq \beta_{\varepsilon, N}(0) \leq \beta_{\varepsilon}(0) .
$$

Next, if $x^{0} \in \Omega$ then, since $u_{e, N}^{n+1}-q$ takes its maximum at $x^{0}$,

$$
L\left(u_{\varepsilon, N}^{n+1}-q\right) \geq 0 \text { at } x^{0} .
$$


Then, using (2.6) and (2.4),

$$
\xi\left(x^{0}\right) \leq f\left(x^{0}, u^{n}\left(x^{0}\right)\right)+L q\left(x^{0}\right) \leq c .
$$

So that

$$
\left|\beta_{\varepsilon, N}\left(u_{\varepsilon, N}^{n+1}-q\right)\right| \leq c \text { independently of } \varepsilon, N, n .
$$

By $L^{p}$ estimates we conclude from (2.6)

$$
\left\|u_{\varepsilon, N}^{n+1}\right\|_{2, p}<c \quad \text { independently of } \varepsilon, N, n .
$$

Taking $N$ sufficiently large, we see that $u_{\varepsilon, N}^{n+1}$ is a solution of the problem (2.5), i.e., $u_{\varepsilon, N}^{n+1}=u_{\varepsilon}^{n+1}$. Letting $\varepsilon \rightarrow 0$, we complete the proof of (2.4).

As $\varepsilon=\varepsilon_{m} \rightarrow 0$ and $n \rightarrow \infty$, we have

$$
\begin{gathered}
u_{\varepsilon}^{n} \rightarrow u^{n} \quad \text { weakly in } W^{2, p}(\Omega), \text { uniformly in } \bar{\Omega}, \\
u^{n} \rightarrow u \quad \text { weakly in } W^{2, p}(\Omega), \text { uniformly in } \bar{\Omega} .
\end{gathered}
$$

Observe, that in both cases we have convergence of the full sequence, not just for a subsequence, because $u^{n}$ is the unique solution of (2.2), and $\left\{u^{n}\right\}$ a monotone sequence. Now we pass to the limit in (2.5) along the diagonal sequence as $\varepsilon=\varepsilon_{m} \rightarrow 0, n \rightarrow \infty$. From (2.7) it follows that $u \leq q(x)$ in $\Omega$. Also, notice that

$$
\begin{aligned}
& \beta_{\varepsilon}\left(u_{\varepsilon}^{n+1}-q\right) \rightarrow 0 \text { on the set }\{x \mid u(x)<q(x)\}, \\
& \varliminf_{\varepsilon \rightarrow 0, n \rightarrow \infty} \beta_{\varepsilon}\left(u_{\varepsilon}^{n+1}-q\right) \geq 0 .
\end{aligned}
$$

It follows that $u(x)$ is a solution of (2.11). Observe, that here it is crucial that we have $u^{n} \rightarrow u$ for a full sequence.

Finally, since $f\left(x, u^{n}\right) \in C^{\alpha}(\bar{\Omega})$, it follows from [6, p. 31] that $u \in W_{l o c}^{2, \infty}(\Omega)$.

REMARK 1. Similarly to the above, if we start with $u^{0}=\varphi$, then the sequence $\left\{u^{n}(x)\right\}$, defined by (2.2), will be increasing in $n$, and converge to a solution of $(2.1)$.

REMARK 2. The solution obtained in the theorem and the one from the previous remark are respectively the maximal and the minimal solutions of (2.1). Indeed, if $T$ is the map $u^{n} \rightarrow u^{n+1}$, defined by (2.2), and $v$ is any solution of (2.1), then $v \leq u^{0}=q, T v=v \leq T u^{0}=u^{1}$, and so on ( $T$ is monotone).

REMARK 3. Let $u_{1}$ denote the maximal solution of the problem (2.1) with $q_{1}(x)$ in place of $q(x)$ and $f_{1}(x, u)$ in place of $f(x, u), q_{1}(x) \geq q(x), f_{1}(x, u)$ $\geq f\left(x, u^{n}\right)$ for all $x \in \Omega$ and $\varphi(x) \leq u \leq q_{1}(x)$. Then $u_{1}(x) \geq u(x)$. The proof is similar to that of the previous remark. 
REMARK 4. It is easy to generalize the theorem to allow less restrictive regularity assumptions for the obstacle. Namely, it is enough to assume that

$$
\begin{gathered}
q \in C^{0,1}(\Omega), \\
\partial^{2} q / \partial \xi^{2} \leq c \quad \text { in } \mathscr{D}^{\prime}\left(\Omega_{0}\right), \text { for any direction } \xi,
\end{gathered}
$$

where $\Omega_{0}$ is a neightorhood of $\bar{\Omega}$ (sce Theorem 1.3 .5 in [6]).

REMARK 5. One checks that the theorem 1 holds if $q(x) \in W^{2, p}(\Omega)$ with $p>n$. Solution $u$ is then in $W^{2 \cdot p}(\Omega)$ too. (See the problem 1 on p. 29 in [6]).

THEOREM 2. In addition to the conditions of the Theorem 1 assume that $f(x, u)=u r(x, u)$, where $r(x, u)$ is strictly decreasing in $u$ for $x \in \Omega, u>0$. Also, assume that $L u$ is of the form $L u=-\sum_{i, j=1}^{n} \frac{\partial}{\partial x_{i}}\left(a_{i j}(x) \frac{\partial u}{\partial x_{j}}\right)+c u$ with $a_{i j} \in C^{1}(\Omega), c \in C^{0}(\Omega), a_{i j}=a_{j i}$ for all $i$ and $j$. Then the problem (2.1) can have at most one positive in $\Omega$ solution of class $W^{2, p}(\Omega), p \geq 2$.

Proof. Let $u(x)$ be the solution of $(2.1)$ produced in the theorem 1 , and $w(x)$ any other solution of (2.1). Then $u(x) \geq w(x)$. Define $A=\{x \in$ $\Omega \mid w(x)<u(x)\}$, and notice that $L w=f(x, w)$ in $A$. Notice also that at $\partial \Omega$ the conormal vector points outside of $\Omega$, and hence the conormal derivative of $u$ is not greater than that of $w$. Then

$$
\begin{aligned}
0 & \leq \int_{\partial \Omega} g\left(\frac{\partial w}{\partial \nu_{L}}-\frac{\partial u}{\partial \nu_{L}}\right)=\int_{\Omega} w L u-u L w=\int_{A} w L u-u L w \\
& \leq \int_{A} u w(r(x, u)-r(x, w)) \leq 0,
\end{aligned}
$$

and hence the set $A$ is empty, i.e., $u=w$.

REMARK. Theorem 1 and 2 are applied in the next section to a population model, where it is possible to establish existence of a subsolution $\varphi(x)>0$.

Next we discuss regularity of the free boundary. Denote $\Lambda=\{x \in$ $\Omega \mid u(x)=q(x)\}, N=\{x \in \Omega \mid u(x)<q(x)\}, \Gamma=\partial N$. For $x_{0} \in \Gamma, B_{r}$ (or $B_{r}\left(x_{0}\right)$ ) denotes the ball of radius $r$ around $x_{0} ;|\cdot|$ denotes the Lebesque measure of a set.

THEOREM 3. In the conditions of the Theorem 1 assume that at some point $x_{0} \in \Gamma$ we have $\lim \sup _{r \rightarrow 0} \frac{\left|\Lambda \cap B_{r}\right|}{\left|B_{r}\right|}>0$, and

$$
-L q\left(x_{0}\right)+f\left(x_{0}, q\left(x_{0}\right)\right)>0 .
$$


Assume also that $q \in C^{3}, f \in C^{1}$ in a neighborhood of $x_{0}$. Then $u \in$ $C^{2}\left((N \cup \Gamma) \cap B_{r}\right)$ for some $r>0$ and $\Gamma \in C^{1+\alpha}$ for any $0<\alpha<1$ in a neighborhood of $x_{0}$. Furthermore if $f \in C^{m+\beta}$ in both arguments ( $m=$ integer $\geq$ $1,0<\beta<1)$ then $\Gamma \in C^{m+\beta+1}$. Finally if $f$ is analytic so is $\Gamma$.

Proof. Define $v=q-u$. Then from (2.1), $v \geq 0$ and

$$
L v-L q+f(x, q-v) \geq 0 \text { in } \Omega \text {. }
$$

At $x_{0},-L q+f(x, q-v)=-L q\left(x_{0}\right)+f\left(x_{0}, q\left(x_{0}\right)\right)>0$, and hence by the Caffarelli's theorem [5], $u \in C^{2}\left((N \cup \Gamma) \cap B_{r}\right)$ for some $r>0$ and $\Gamma \in C^{1}$ in some neighborhood of $x_{0}$. Then our assertions follow by the Theorem $1^{\prime}$ in [10].

Next we give a useful condition for (2.9) to hold, in case $L=-\Delta$.

Proposition. For the problem (2.1) assume that $f(x, q)+\Delta q$ and $\nabla(f(x, q)+$ $\Delta q)$ do not vanish simultaneously at $x_{0} \in \Gamma ; f$ in increasing in $u, q \in C^{3}$ and $f \in C^{1}$ in a neighborhood of $x_{0}$. Then (2.9) holds.

Proof. From (2.1), $\psi=\Delta q+f(x, q) \geq 0$ a.e. on $\Gamma$. By continuity this inequality holds everywhere on $\Gamma$. We show that $\psi>0$ on $\Gamma$, following the proof of the lemma 7.3 in [6, p. 195]. Suppose that $\psi\left(x_{0}\right)=0$. Since $\nabla \psi\left(x_{0}\right) \neq 0$, then for any small $\varepsilon>0$ one can find a small $R>0$ such that

$$
\psi<0 \text { in } K_{\varepsilon} \cap B_{R}\left(x_{0}\right),
$$

where $K_{\varepsilon}$ is the cone $K_{\varepsilon}=\left\{-\left(x-x_{0}, \nabla \psi\left(x_{0}\right)\right) \geq \varepsilon\left|x-x_{0}\right|\right\}$. The function $v=q-u$ then satisfies $v \geq 0, v\left(x_{0}\right)=0, \nabla v\left(x_{0}\right)=0$,

$$
-\Delta v=-\Delta q+\Delta u>f(x, q)-f(x, u) \geq 0 \text { in } K_{\varepsilon} \cap B_{R}\left(x_{0}\right),
$$

and then one gets the same contradiction as in [6].

\section{Logistic population model with diffusion and an obstacle to growth}

Let $u(x, t)>0$ denote the population density of a certain species at the position $x \in \Omega$ and time $t>0$, which obeys the logistic growth model with diffusion:

$$
u_{t}-\Delta u=u(a-u) \quad \text { in } \Omega, \quad u=0 \text { on } \partial \Omega .
$$

Here the function $a(x)$ represents the rate of reproduction, and the boundary condition $u=0$ can be interpreted as a "hostile environment", or "emigration of species". 
As a first step in the analysis of (3.1), one usually considers the steady state problem

$$
\Delta u+u(a-u)=0 \quad \text { in } \Omega, \quad u=0 \text { on } \partial \Omega \text {. }
$$

We recall ([11]) that for $a \leq \lambda_{1}$ the problem (3.2) has no positive solutions, whiie for $a>\lambda_{1}$ there exists a uniquie positive solution $u_{a}$ (with $\left(a-\lambda_{i}\right) \varphi_{\mathrm{i}} \leq$ $u_{a}<a$ in case $a=$ constant). Moreover, $u_{a}$ is increasing in $a$.

Now we assume that there is an obstacle to growth of the species, i.e., a function $q(x) \in C^{2}(\bar{\Omega}), q(x)>0$ in $\Omega$, such that $u(x) \leq q(x)$ in $\Omega$, and look for a steady-state solution. The interesting case is, of course, when the obstacle $q(x)$ interferes with the "natural" steady state, i.e., the set $\{x \in$ $\left.\Omega: q(x)<u_{a}\right\}$ is non-empty. In the coincidence set $\{u=q(x)\}$ we require that $-\Delta u \leq u(a-u)$, for otherwise $u$ cannot be a steady state solution of (3.1). (If this inequality was violated at some point, the population would instantaneously decrease at that point.) So that we are looking for $u(x)>0$, satisfying the following variational inequality:

$$
\begin{gathered}
-\Delta u \leq u(a-u) \quad \text { in } \Omega \\
(\Delta u+u(a-u))(u-q)=0 \quad \text { in } \Omega \\
u \leq q \text { in } \Omega, u=0, \quad \text { on } \partial \Omega .
\end{gathered}
$$

Proposition. If $a \leq \lambda_{1}$, the problem (3.3) has no positive solution. If $a>\lambda_{1}$, $a \in C^{\alpha}(\bar{\Omega})$ and $q(x)>\delta \varphi_{1}$ for some $\delta>0$, then the problem (3.3) has a unique positive solution of class $W^{2, p}(\Omega) \cap W_{\text {loc }}^{2, \infty}(\Omega)$ for any $p \geq 2$, which is increasing in $a$.

Proof. Multiply the first equation in (3.3) by $u>0$ and integrate by parts. We get:

$$
-\int_{\Omega}|\nabla u|^{2}+\int_{\Omega} a u^{2}-\int_{\Omega} u^{3} \geq 0,
$$

and the non-existence for $a \leq \lambda_{1}$ follows from the inequality $\int_{\Omega}|\nabla u|^{2} \geq$ $\lambda_{1} \int_{\Omega} u^{2}$.

In the case $a>\lambda_{1}$, existence follows from the Theorem 1 (notice that $\delta \varphi_{1}$ is a subsolution for $\delta$ sufficiently small). Uniqueness follows by the Theorem 2. Monotone dependence on $a(x)$ follows by the Remark 3 to the Theorem 1. 
REMARK. By the Theorem 3 we can conclude that the free boundary is as smooth as the function $a(x)$, provided it is known to be Lipschitz continuous.

\section{Monotone Newton's method}

In this section we present a Newton-like method with monotone convergence, first for semilinear elliptic problems and then for our obstacle problem (2.1). Throughout the section we assume that $L$ is a uniformly elliptic operator of the form

$$
L u=-\sum_{i, j=1}^{n} \frac{\partial}{\partial x_{i}}\left(a_{i j} \frac{\partial u}{\partial x_{j}}\right)+c(x) u,
$$

with $a_{i j} \in C^{1+\alpha}(\bar{\Omega}), c(x) \in C^{\alpha}(\bar{\Omega}), c(x) \geq 0$ for $x \in \bar{\Omega}, g(x) \in C^{2+\alpha}(\bar{\Omega}), \Omega$ bounded domain in $R^{n}$ with $C^{2+\alpha}$ boundary, $0<\alpha<1$.

TheOREM 4. Consider the problem

$$
\begin{gathered}
L u=f(x, u) \quad \text { in } \Omega \\
u=g(x) \quad \text { on } \partial \Omega
\end{gathered}
$$

In addition to the conditions above assume that $g(x) \geq 0$, and the following.

(i) There exists a function $\varphi(x)>0$ in $\Omega$ and $\varphi(x)=0$ on $\partial \Omega, \varphi(x) \in$ $C^{2}(\Omega)$, called subsolution, which satisfies

$$
\begin{gathered}
L \varphi \leq f(x, \varphi) \quad \text { in } \Omega \\
\varphi \leq g(x) \text { on } \partial \Omega,
\end{gathered}
$$

and a supersolution $u_{0}(x) \in C^{2}(\Omega)$, which satisfies (4.3) with the inequality signs reversed. Moreover $u_{0}(x) \geq \varphi(x)$ in $\Omega, u_{0}(x)>0$ in $\bar{\Omega}$.

(ii) For $x \in \Omega$ and $\varphi(x) \leq u \leq u_{0}(x)$ the function $f$ is concave down in $u$, and of the form $f(x, u)=u r(x, u)$ where $r(x, u)$ is strictly decreasing in $u$.

(iii) $f, f_{u x} \in C^{0}$ in $x$ and $u$, and $f \in C^{2}$ in $u$ for $x \in \bar{\Omega}$ and $\varphi(x) \leq u \leq$ $u_{0}(x)$. Then the unique positive solution of (4.2) can be obtained as a limit of the sequence $\left\{u_{n}(x)\right\}$, defined by solving (starting with the supersolution $u_{o}(x)$ )

$$
\begin{gathered}
L u_{n+1}=f\left(x, u_{n}\right)+f_{u}\left(x, u_{n}\right)\left(u_{n+1}-u_{n}\right) \text { in } \Omega \\
u_{n+1}=g(x) \text { on } \partial \Omega .
\end{gathered}
$$

Moreover, the speed of convergence is quadratical.

Proof. First we notice that the problem (4.2) has a unique positive solution of class $C^{2}(\Omega)$. Existence is standard, see e.g., [2], where it is shown that there exists a maximal solution $w$ in the order interval $\left[\varphi, u_{0}\right]$, i.e., for any other 
solution $u$ in the same order interval, one has $u \leq w$. Notice that at $\partial \Omega$ the conormal vector $\nu_{L}=\left(\nu_{L 1}, \ldots, \nu_{L n}\right), \nu_{L i}=\sum_{j=1}^{n} a_{i j} \nu_{j}$ (with $\nu=\left(\nu_{1}, \ldots, \nu_{n}\right)$ denoting the normal vector) points outside of $\Omega$, and hence the conormal derivative of $w$ is not greater than that of $u$. Then

$$
\begin{aligned}
0 & \leq \int_{\partial \Omega} w \frac{\partial u}{\partial \nu_{L}}-u \frac{\partial w}{\partial \nu_{L}}=\int_{\Omega} u L w-w L u \\
& =\int_{\Omega} u w(r(x, w)-r(x, u)) \leq 0,
\end{aligned}
$$

which implies that $u=w$. Hence we have uniqueness in the order interval $\left[\varphi, u_{0}\right]$. Uniqueness for (4.2) will then follow from the observation that 0 and $\lambda u_{0}$ are respectively sub- and supersolutions for any $\lambda>1$.

So let $u(x)$ denote the unique positive solution of (4.2). We shall prove inductively the following statements.

(i) The function $u_{n}(x)$ is a supersolution of both (4.2) and (4.4) for all $n \geq 0$. Indeed, by concavity

$$
L u_{n+1}=f\left(x, u_{n}\right)+f_{u}\left(x, u_{n}\right)\left(u_{n+1}-u_{n}\right) \geq f\left(x, u_{n+1}\right),
$$

establishing the first part of the claim. Then, the function $u_{n}(x)$ is a supersolution of (4.4).

(ii) $\varphi$ is a subsolution of (4.4) for all $n \geq 0$. Indeed

$$
L \varphi \leq f(x, \varphi) \leq f\left(x, u_{n}\right)+f_{u}\left(x, u_{n}\right)\left(\varphi-u_{n}\right) .
$$

(iii) The problem (4.4) can have at most one solution. If there are two solutions of (4.4), then their difference $z$ satisfies

$$
\begin{aligned}
L z-f_{u}\left(x, u_{n}\right) z & =0 & & \text { in } \Omega \\
z & =0 & & \text { on } \partial \Omega .
\end{aligned}
$$

Let $\bar{u}$ denote the unique positive solution of

$$
\begin{aligned}
L \bar{u} & =f(x, \bar{u}) \quad \text { in } \Omega \\
\bar{u} & =0 \quad \text { on } \partial \Omega .
\end{aligned}
$$

Claim: $\bar{u} \leq u_{n}$ for any $n \geq 0$. Indeed, $u_{n}$ is a supersolution of (4.6) for all $n$, while $\varphi$ is a subsolution. Hence there exists a solution of (4.6) in the order interval $\left[\varphi, u_{n}\right]$, which by uniqueness is $\bar{u}$.

From (4.6) we conclude that the principal eigenvalue of the problem

$$
\begin{aligned}
L v-r(x, \bar{u}) v & =\lambda v & & \text { in } \Omega \\
v & =0 & & \text { on } \partial \Omega
\end{aligned}
$$


is zero, and the principal eigenfunction is $\bar{u}>0$. (Recall that only the principal eigenfunction of the uniformly elliptic operator does not change sign.) By our assumptions and since $\bar{u} \leq u_{n}$, it follows that

$$
\begin{gathered}
f_{u}(x, u)=r(x, u)+u r_{u}(x, u)<r(x, u), \\
f_{u}\left(x, u_{n}\right)<r\left(x, u_{n}\right) \leq r(x, \bar{u}) .
\end{gathered}
$$

Hence, the principal eigenvalue of

$$
L z-f_{u}\left(x, u_{n}\right) z=\lambda z \quad \text { in } \Omega, \quad z=0, \quad \text { on } \partial \Omega
$$

is positive, and so (4.5) has only the trivial solution $z=0$.

$$
\varphi \leq u \leq u_{n+1} \leq u_{n} \text { for all } n \geq 0 .
$$

The problem (4.4) has a unique solution $u_{n+1}$ in the order interval $\left[\varphi, u_{n}\right]$ (existence again follows by the standard method of super- and subsolutions). Since $u_{n+1}$ is a supersolution of (4.2), it follows that there exists a solution of (4.2) in the order interval $\left[\varphi, u_{n+1}\right]$, which by uniqueness has to be $u(x)$ (so that $\varphi \leq u \leq u_{n+1}$ ).

We have thus produced a sequence $\left\{u_{n}(x)\right\}$ with $\varphi \leq u \leq \cdots \leq u_{n} \leq \cdots \leq$ $u_{2} \leq u_{1} \leq u_{0}$. It is clear that $w(x)=\lim _{n \rightarrow \infty} u_{n}(x)$ exists. It will follow that $w=u$, once we show that $w(x)$ is a solution of (4.2). Write

$$
\begin{aligned}
L\left(u_{n+1}-u_{n}\right)= & f\left(x, u_{n}\right)-f\left(x, u_{n-1}\right)+f_{u}\left(x, u_{n}\right)\left(u_{n+1}-u_{n}\right) \\
& -f_{u}\left(x, u_{n-1}\right)\left(u_{n}-u_{n-1}\right) \text { in } \Omega \\
& u_{n+1}-u_{n}=0 \text { on } \partial \Omega .
\end{aligned}
$$

Since $f_{u}(x, u)$ is bounded for $x \in \bar{\Omega}, \varphi \leq u \leq u_{0}$, it follows that the right hand side of (4.8) tends to zero in $L^{p}(\Omega)$, for any $p>1$. It follows by the standard elliptic theory (see e.g., [1]) that $u_{n+1}-u_{n} \rightarrow 0$ in $W^{2, p}(\Omega)$, and then by the Sobolev's imbedding theorem, $u_{n+1}-u_{n} \rightarrow 0$ in $C^{\alpha}(\Omega)$ for any $0<\alpha<1$. Hence, $w \in C^{\alpha}$ and $\left\|u_{n}\right\|_{C^{\alpha}}<c$ uniformly in $n$. Once easily sees that under our assumptions

$$
\begin{aligned}
\left\|f_{u}\left(x, u_{n}\right)\right\|_{C^{\alpha}} & \leq c\left(\left\|f_{u x}\left(x, u_{n}\right)\right\|_{C^{\circ}}+\left\|f_{u u}\left(x, u_{n}\right)\right\|_{C^{\circ}}\left\|u_{n}\right\|_{C^{\alpha}}+\left\|f_{u}\left(x, u_{n}\right)\right\|_{C^{\circ}}\right) \\
& \leq c
\end{aligned}
$$

Writing

$$
f\left(x, u_{n}\right)-f\left(x, u_{n-1}\right)=\int_{0}^{1} f_{u}\left(x, t u_{n}+(1-t) u_{n-1}\right) d t\left(u_{n}-u_{n-1}\right),
$$

using the inequality $\|u v\|_{C^{\alpha}} \leq 3\|u\|_{C^{a}}\|v\|_{C^{a}}$ and (4.9), we see that the right hand side of (4.8) tends to zero in $C^{\alpha}(\Omega)$. By the Schauder's estimates it 
follows that $u_{n+1}-u_{n} \rightarrow 0$ in $C^{2+\alpha}(\Omega)$. Hence $w \in C^{2+\alpha}$, and we can pass to the limit in (4.4), concluding that $w(x)$ is a solution of (4.2).

It remains to show that the convergence of $\left\{u_{n}(x)\right\}$ is quadratic. Write

$$
\begin{aligned}
& L\left(u_{n+1}-u_{n}\right)-f_{u}\left(x, u_{n}\right)\left(u_{n+1}-u_{n}\right)= f\left(x, u_{n}\right)-f\left(x, u_{n-1}\right) \\
&-f_{u}\left(x, u_{n-1}\right)\left(u_{n}-u_{n-1}\right) \\
& \equiv \bar{f} \text { in } \Omega \\
& u_{n+1}-u_{n}=0 \quad \text { on } \partial \Omega .
\end{aligned}
$$

Since $\bar{f}=(1 / 2) f_{u u}\left(x, \theta u_{n}+(1-\theta) u_{n-1}\right)\left(u_{n}-u_{n-1}\right)^{2}$ for some $0<\theta<1$, and for fixed $\bar{f}$ there exists a unique solution $u_{n+1}-u_{n}$ of (4.10) (uniqueness is proved similarly to (4.4)), it follows by the $L^{p}$ estimates and imbedding theorems (for $p>n$ ),

$$
\begin{aligned}
\left\|u_{n+1}-u_{n}\right\|_{C^{\circ}} & \leq c\left\|u_{n+1}-u_{n}\right\|_{W^{2, p}} \leq c\|\bar{f}\|_{L p} \\
& \leq c\left\|u_{n}-u_{n-1}\right\|_{C^{\circ}}^{2} \quad(c \text { is independent of } n) .
\end{aligned}
$$

Remark. A similar result for the logistic ODE appears in [12].

Next we take up the more involved case of the obstacle problem.

THEOREM 5. Consider the variational inequality (2.1). Assume that $L, f(x, u)$, $g(x)$ and $\Omega$ satisfy the same conditions as in the theorem 4 with $f$ being strictly concave in $u$, and there exists a subsolution $\varphi(x)>0$ in $\Omega$ and $\varphi(x)=0$ on $\partial \Omega$, defined as a $C^{2}(\Omega)$ function satisfying (4.3) and $\varphi(x)<q(x)$ in $\Omega$. We also assume that $f_{u}(x, u) \leq 0$ for $\varphi \leq u \leq q$ and $x \in \Omega$, and that $q(x) \in C^{2}(\Omega)$, $g(x)<q(x)$ for $x \in \bar{\Omega}$. Then the unique positive solution of $(2.1)$ which is of class $W^{2, p}(\Omega)$ for any $p>1$, can be obtained as a limit of the sequence $\left\{u^{n}(x)\right\}$, defined by letting $u^{0}=q$ and solving for $n \geq 0$

$$
\left.\begin{array}{rl}
L u^{n+1} & \leq f\left(x, u^{n}\right)+f_{u}\left(x, u^{n}\right)\left(u^{n+1}-u^{n}\right) \\
u^{n+1} & \leq u^{n} \\
{\left[L u^{n+1}\right.} & \left.-f\left(x, u^{n}\right)-f_{u}\left(x, u^{n}\right)\left(u^{n+1}-u^{n}\right)\right]\left(u^{n+1}-u^{n}\right)=0
\end{array}\right\} \quad \text { a.e. in } \Omega
$$

Moreover, the speed of convergence is quadratical.

Proof. Existence and uniqueness of positive solution of (2.1) follows by the Theorems 1 and 2. (Solution $u^{n}$ is in $W^{2, p}(\Omega)$. By our Remark 5 to the theorem 1, the iterations in (4.11) can be continued for all $n$.) Notice next that $\varphi(x)$ is a subsolution of (4.11) for any $n \geq 0$. Indeed, $\varphi(x) \leq g(x)$ on $\partial \Omega, \varphi(x)<q(x)$ in $\Omega$, and by concavity

$$
L \varphi \leq f(x, \varphi) \leq f\left(x, u^{n}\right)+f_{u}\left(x, u^{n}\right)\left(\varphi-u^{n}\right) .
$$


Applying the Theorem 1 to (4.11) with

$$
\bar{f}\left(x, u^{n+1}\right) \equiv f\left(x, u^{n}\right)+f_{u}\left(x, u^{n}\right)\left(u^{n+1}-u^{n}\right)
$$

in place of $f(x, u)$, we see that the problem (4.11) has a positive solution. Next we claim that the positive solution of (4.11) is unique. Indeed, (4.11) is an obstacle problem for the linear operator $L-f_{u}\left(x, u^{n}\right)$ with the forcing term $f\left(x, u^{n}\right)-f_{u}\left(x, u^{n}\right) u^{n}$, so that uniqueness follows by the standard result (see e.g., [6, p. 26]).

We have produced a sequence of iterates $\left\{u^{n}(x)\right\}$ with

$$
\varphi \leq \cdots \leq u^{n+1} \leq u^{n} \leq \cdots \leq u^{1} \leq q .
$$

Call $w(x)=\lim _{n \rightarrow \infty} u^{n}(x)$. We proceed to show that $w(x)$ is a solution of (4.11). By uniqueness it will follow that $w=u$. To pass to the limit we need to estimate $\left\|u^{n}(x)\right\|_{2, p}$ independently of $n$. It is not clear how to get such an estimate from (4.11), because the obstacle depends on $n$. To overcome this difficulty we now introduce an equivalent auxiliary problem. Starting with $\bar{u}^{0}=q$, define a sequence of iterates by solving $(n=0,1, \ldots)$

$$
\left.\begin{array}{l}
L \bar{u}^{n+1} \leq f\left(x, \bar{u}^{n}\right)+f_{u}\left(x, \bar{u}^{n}\right)\left(\bar{u}^{n+1}-\bar{u}^{n}\right) \\
\bar{u}^{n+1} \leq q(x) \\
\left(L \bar{u}^{n+1}-f\left(x, \bar{u}^{n}\right)-f_{u}\left(x, \bar{u}^{n}\right)\left(\bar{u}^{n+1}-\bar{u}^{n}\right)\right)\left(\bar{u}^{n+1}-q\right)=0
\end{array}\right\}
$$

Existence and uniqueness of solution of $(4.11)^{\prime}$ follows in the same way as for (4.11).

We claim that $\bar{u}^{n}=u^{n}$ for all $n \geq 0$. For this it suffices to show that $u^{n+1}<$ $u^{n}$ for $n=1,2, \ldots$, in $\left\{x \in \Omega \mid u^{n}(x)<q(x)\right\}$ (increasing the obstacle away from the contact set does not change solution of the variational inequality (4.11), and by the inductive assumption the problems (4.11) and (4.11) differ only in the obstacle). Assume that on the contrary that $u^{n+1}(x)$ and $u^{n}(x)$ touch away from the obstacle, and let $n \geq 1$ be the first index where this happens, i.e., at some point $x_{0} \in \Omega$ we have $u^{n+1}\left(x_{0}\right)=u^{n}\left(x_{0}\right)$ while $u^{n}\left(x_{0}\right)<$ $q\left(x_{0}\right)$. Then $u^{n}\left(x_{0}\right)<u^{n-1}\left(x_{0}\right)$ (if $n=1$ then obviously $u^{1}\left(x_{0}\right)<q\left(x_{0}\right)=$ $\left.u^{0}\left(x_{0}\right)\right)$. By the first relation of (4.11) and the third one in $(4.11)^{\prime}$ it follows that at $x_{0}$

$$
L\left(u^{n+1}-u^{n}\right) \leq f\left(x, u^{n}\right)-f\left(x, u^{n-1}\right)-f_{u}\left(x, u^{n-1}\right)\left(u^{n}-u^{n-1}\right) .
$$

Since $x_{0}$ is a point of maximum of $u^{n+1}-u^{n}$, it follows that $L\left(u^{n+1}-u^{n}\right) \geq 0$ at $x_{0}$, while the right hand side of (4.12) is negative by the strict concavity of $f$, a contradiction. This proves our claim, and in particular that $w(x)=$ $\lim _{n \rightarrow \infty} \bar{u}^{n}(x)$. 
Examining the proof of the Theorem 1, one sees that the $W^{2, p}(\Omega)$ norm of the solution of $(4.11)^{\prime}$ is bounded independently of $n$ (it depends only on $|q|_{C^{2},} \max _{x \in \bar{\Omega}, \varphi \leq u \leq q} \bar{f}(x, u),|g|_{W^{2, p},}$ and $\left.p\right)$. Since $u^{n}=\bar{u}^{n}$, it follows that for any $p>1$

$$
\left\|u^{n}\right\|_{W^{2, p}} \leq c \text { independently of } n .
$$

We now pass to the limit in (4.i i $)^{\prime}$, dropping the bars for corivenience.

Recall that $u^{n+1}$ can be obtained as a limit when $\varepsilon \rightarrow 0$ of the solution $u_{\varepsilon}^{n+1}$ of the penalized problem (with $\beta_{\varepsilon}(t)$ as in the Theorem 1)

$$
\begin{gathered}
L u_{\varepsilon}^{n+1}-f_{u}\left(x, u^{n}\right) u_{\varepsilon}^{n+1}+\beta_{\varepsilon}\left(u_{\varepsilon}^{n+1}-q\right)=f\left(x, u^{n}\right)-f_{u}\left(x, u^{n}\right) u^{n} \\
u_{\varepsilon}^{n+1}=g(x) \text { in } \Omega \\
\end{gathered}
$$

(We are applying standard theory to the operator $L-f_{u}\left(x, u^{n}\right)$ in place of $L$. Recall that $f_{u}\left(x, u^{n}\right) \in C^{\alpha}(\bar{\Omega})$.) Recall also that to solve (4.14) one introduces $\beta_{\varepsilon, N}(t)=\max \left\{\min \left(\beta_{\varepsilon}(t), N\right),-N\right\}$, and considers the problem

$$
\begin{array}{cc}
L u_{\varepsilon, N}^{n+1}-f_{u}\left(x, u^{n}\right) u_{\varepsilon, N}^{n+1}+\beta_{\varepsilon, N}\left(u_{\varepsilon, N}^{n+1}-q\right)=f\left(x, u^{n}\right)-f_{u}\left(x, u^{n}\right) u^{n} & \text { in } \Omega \\
u_{\varepsilon, N}^{n+1}=g & \text { on } \partial \Omega .
\end{array}
$$

We need a bound on the function $\xi(x)=\beta_{\varepsilon, N}\left(u_{\varepsilon, N}^{n+1}-q\right)$. By the definition of $\beta_{\varepsilon}, \xi(x) \geq-c$. Let now $x_{0}$ be the point of maximum of $\xi(x)$. We may assume that $\xi\left(x_{0}\right)>\beta_{\varepsilon}(0)$ for $0<\varepsilon<1$ (otherwise we have the desired bound on $\xi(x))$. Notice that $x_{0} \notin \partial \Omega$, for otherwise

$$
\xi\left(x_{0}\right)=\beta_{\varepsilon, N}(g-q) \leq \beta_{\varepsilon, N}(0) \leq \beta_{\varepsilon}(0),
$$

Since $u_{\varepsilon, N}^{n+1}-q$ takes its maximum at $x_{0} \in \Omega$,

$$
L\left(u_{\varepsilon, N}^{n+1}-q\right) \geq 0 \text { at } x_{0} .
$$

By (4.15) and our assumptions of $f$

$$
\begin{aligned}
\xi\left(x_{0}\right) & \leq f\left(x_{0}, u^{n}\left(x_{0}\right)\right)-f_{u}\left(x_{0}, u^{n}\left(x_{0}\right)\right) u^{n}\left(x_{0}\right)-L q\left(x_{0}\right) \\
& +f_{u}\left(x_{0}, u^{n}\left(x_{0}\right)\right) u^{n+1}\left(x_{0}\right) \leq c .
\end{aligned}
$$

So that

$$
\left|\beta_{\varepsilon, N}\left(u_{\varepsilon, N}^{n+1}-q\right)\right| \leq c \quad \text { independently of } \varepsilon, N, n .
$$

By the $L^{p}$ estimates we conclude from (4.15)

$$
\left\|u_{\varepsilon, N}^{n+1}\right\|_{2, p} \leq c \text { independently of } \varepsilon, N, n .
$$

Taking $N$ sufficiently large we see that $u_{\varepsilon, N}^{n+1}$ is a solution of the problem (4.14), i.e., $u_{\varepsilon, N}^{n+1}=u_{\varepsilon}^{n+1}$. 
As $\varepsilon \rightarrow 0$ (along some sequence $\left\{\varepsilon_{m}\right\}$ ) and $n \rightarrow \infty$, we have

$$
\begin{aligned}
& u_{\varepsilon}^{n} \rightarrow u^{n} \quad \text { weakly in } W^{2, p}(\Omega) \text {, uniformly in } \bar{\Omega} \\
& u^{n} \rightarrow w \quad \text { weakly in } W^{2, p}(\Omega), \text { uniformly in } \bar{\Omega} .
\end{aligned}
$$

Observe that by monotonicity of the sequence $\left\{u^{n}(x)\right\}$ we have convergence for the full sequence. Now we pass to the limit in (4.14) along a diagonal sequence as $\varepsilon=\varepsilon_{m} \rightarrow 0, n \rightarrow \infty$. From (4.16) it follows that $w \leq q(x)$ in $\Omega$. Also, notice that

$$
\begin{gathered}
\beta_{\varepsilon}\left(u_{\varepsilon}^{n+1}-q\right) \rightarrow 0 \text { on the set }\{x \mid w(x)<q(x)\} \\
\varliminf_{\varepsilon \rightarrow 0, n \rightarrow \infty} \beta_{\varepsilon}\left(u_{\varepsilon}^{n+1}-q\right) \geq 0 .
\end{gathered}
$$

It follows that $w(x)$ is a solution of (2.1).

Turning to the quadratic convergence, we introduce the bilinear form

$$
a(u, v)=\int_{\Omega}\left(\sum_{i, j=1}^{n} a_{i j}(x) \frac{\partial u}{\partial x_{j}} \frac{\partial v}{\partial x_{i}}+c(x) u v\right),
$$

and the set

$$
K=\left\{v \in H^{1}(\Omega), v-g \in H_{0}^{1}(\Omega), v \leq q \text { a.e. in } \Omega\right\} .
$$

Then the variational inequality $(4.11)^{\prime}$ can be written in the form (dropping bars)

$$
\begin{gathered}
a\left(u^{n+1}, v-u^{n+1}\right)-\int_{\Omega} f_{u}\left(x, u^{n}\right) u^{n+1}\left(v-u^{n+1}\right) \\
\geq \int_{\Omega}\left[f\left(x, u^{n}\right)-f_{u}\left(x, u^{n}\right) u^{n}\right]\left(v-u^{n+1}\right)
\end{gathered}
$$

for any $v \in K$ (see [6]). Similarly

$$
\begin{aligned}
& a\left(u^{n}, v-u^{n}\right)-\int_{\Omega} f_{u}\left(x, u^{n-1}\right) u^{n}\left(v-u^{n}\right) \\
& \quad \geq \int_{\Omega}\left[f\left(x, u^{n-1}\right)-f_{u}\left(x, u^{n-1}\right) u^{n-1}\right]\left(v-u^{n}\right) \text { for any } v \in K .
\end{aligned}
$$

Setting $v=u^{n}$ in (4.17) and $v=u^{n+1}$ in (4.18) and adding the resulting inequalities, we obtain

$$
\begin{aligned}
& -a\left(u^{n+1}-u^{n}, u^{n+1}-u^{n}\right)-\int_{\Omega} f_{u}\left(x, u^{n}\right)\left(u^{n+1}-u^{n}\right)\left(u^{n}-u^{n+1}\right) \\
& \quad \geq-\int_{\Omega}\left[f\left(x, u^{n}\right)-f\left(x, u^{n-1}\right)-f_{u}\left(x, u^{n-1}\right)\left(u^{n}-u^{n-1}\right)\right]\left(u^{n+1}-u^{n}\right) .
\end{aligned}
$$


Since $a(v, v) \geq c\|v\|_{H^{1}}^{2}$ for $v \in H_{0}^{1}$, we obtain using the Schwarz inequality

$$
\left\|u^{n+1}-u^{n}\right\|_{H^{1}(\Omega)} \leq c\left\|\left(u^{n}-u^{n-1}\right)^{2}\right\|_{L^{2}(\Omega)} .
$$

REMARK. Our assumption that $f_{u}(x, u) \leq 0$ is rather restrictive; in particular, it excludes the logistic equation for which $f(x, u)=u(a(x)-u)$. This condition was used to ensure unique solvability of (4.11) and to prove quadratic convergence.

\section{Numerical experiments}

Numerical experiments confirm superiority of the Newton method (as in Theorem 5) to the monotone iteration method (as in Theorem 1). For example, consider the following problem:

$$
\left.\begin{array}{rl}
L u-f(u) & \leq 0 \\
u & \leq 10 \\
(u))(u-10) & =0
\end{array}\right\} \quad \text { a.e. in } \Omega=(-1,1) \times(-1,1)
$$

with

$$
\begin{gathered}
L u=-\left(1+y^{2} x^{2}\right) \partial^{2} u / \partial x^{2}-\left(1+\frac{y}{2}\right) \frac{\partial^{2} u}{\partial y^{2}}+15 u, \\
f(u)=-u^{2}+30 u .
\end{gathered}
$$

At each step an obstacle problem is solved using piecewise linear finite elements. Iterations are performed until supremum norm of the difference between two consecutive iterates becomes less than $\varepsilon=0.005$. Each side of the square is divided into 15 equal pieces. It took only four iterations of the Newton method to achieve the desired accuracy, compared to eleven iterations by the monotone iterations. Result is given in the Table 1 (computations were performed on AMDAHL).

Similar computations were performed in one dimension. For example, solving the obstacle problem for the equation $-u^{\prime \prime}=u(5-u)$ with $0<x<2$, $u(0)=u(2)=0, h=0.1$ and the obstacle $q(x)=(x-1)^{2}+1$, took us 11 iterations and 65 seconds by Picard's iterations on a PC, while only 4 iterations and 41 seconds by Newton's method (calculations were terminated when $\left.\max _{i}\left|u_{n+1}\left(x_{i}\right)-u_{n}\left(x_{i}\right)\right|<10^{-6}\right)$. In the Figure 1 we superimpose the 
TABLE 1

$\begin{array}{lllllllllllllll}0.0 & 0.0 & 0.0 & 0.0 & 0.0 & 0.0 & 0.0 & 0.0 & 0.0 & 0.0 & 0.0 & 0.0 & 0.0 & 0.0 & 0.0\end{array}$ $\begin{array}{lllllllllllllll}0.0 & 1.1 & 2.0 & 2.7 & 3.2 & 3.5 & 3.7 & 3.7 & 3.7 & 3.5 & 3.2 & 2.7 & 2.0 & 1.0 & 0.0\end{array}$ $\begin{array}{lllllllllllllll}0.0 & 2.0 & 2.7 & 5.0 & 5.8 & 6.4 & 6.6 & 6.7 & 6.6 & 6.3 & 5.8 & 4.9 & 4.6 & 2.0 & 0.0\end{array}$ $\begin{array}{lllllllllllllll}0.0 & 2.7 & 5.0 & 6.6 & 7.7 & 8.4 & 8.7 & 8.7 & 8.6 & 8.3 & 7.7 & 6.6 & 4.9 & 2.7 & 0.0\end{array}$ $\begin{array}{lllllllllllllll}0.0 & 3.2 & 5.9 & 7.7 & 8.9 & 9.5 & 9.8 & 9.8 & 9.8 & 9.5 & 8.9 & 7.7 & 5.8 & 3.2 & 0.0\end{array}$ $\begin{array}{lllllllllllllll}0.0 & 3.5 & 6.4 & 8.4 & 9.5 & 10.0 & 10.0 & 10.0 & 10.0 & 10.0 & 9.5 & 8.4 & 6.4 & 3.5 & 0.0\end{array}$ $\begin{array}{lllllllllllllll}0.0 & 3.7 & 6.7 & 8.7 & 9.8 & 10.0 & 10.0 & 10.0 & 10.0 & 10.0 & 9.8 & 8.7 & 6.7 & 3.7 & 0.0\end{array}$ $\begin{array}{lllllllllllllll}0.0 & 3.8 & 6.8 & 8.9 & 9.9 & 10.0 & 10.0 & 10.0 & 10.0 & 10.0 & 9.9 & 8.6 & 6.8 & 3.8 & 0.0\end{array}$ $\begin{array}{lllllllllllllll}0.0 & 4.0 & 7.1 & 9.1 & 10.0 & 10.0 & 10.0 & 10.0 & 10.0 & 10.0 & 10.0 & 9.1 & 7.1 & 4.0 & 0.0\end{array}$ $\begin{array}{lllllllllllllll}0.0 & 4.0 & 7.1 & 9.1 & 10.0 & 10.0 & 10.0 & 10.0 & 10.0 & 10.0 & 10.0 & 9.1 & 7.1 & 4.0 & 0.0\end{array}$ $\begin{array}{lllllllllllllll}0.0 & 4.0 & 7.1 & 9.1 & 10.0 & 10.0 & 10.0 & 10.0 & 10.0 & 10.0 & 10.0 & 9.1 & 7.1 & 4.0 & 0.0\end{array}$ $\begin{array}{lllllllllllllll}0.0 & 4.0 & 7.1 & 9.1 & 10.0 & 10.0 & 10.0 & 10.0 & 10.0 & 10.0 & 10.0 & 9.1 & 7.1 & 4.0 & 0.0\end{array}$ $\begin{array}{lllllllllllllll}0.0 & 43.9 & 7.1 & 9.1 & 10.0 & 10.0 & 10.0 & 10.0 & 10.0 & 10.0 & 10.0 & 9.3 & 7.1 & 4.0 & 0.0\end{array}$ $\begin{array}{lllllllllllllll}0.0 & 0.0 & 0.0 & 0.0 & 0.0 & 0.0 & 0.0 & 0.0 & 0.0 & 0.0 & 0.0 & 0.0 & 0.0 & 0.0 & 0.0\end{array}$

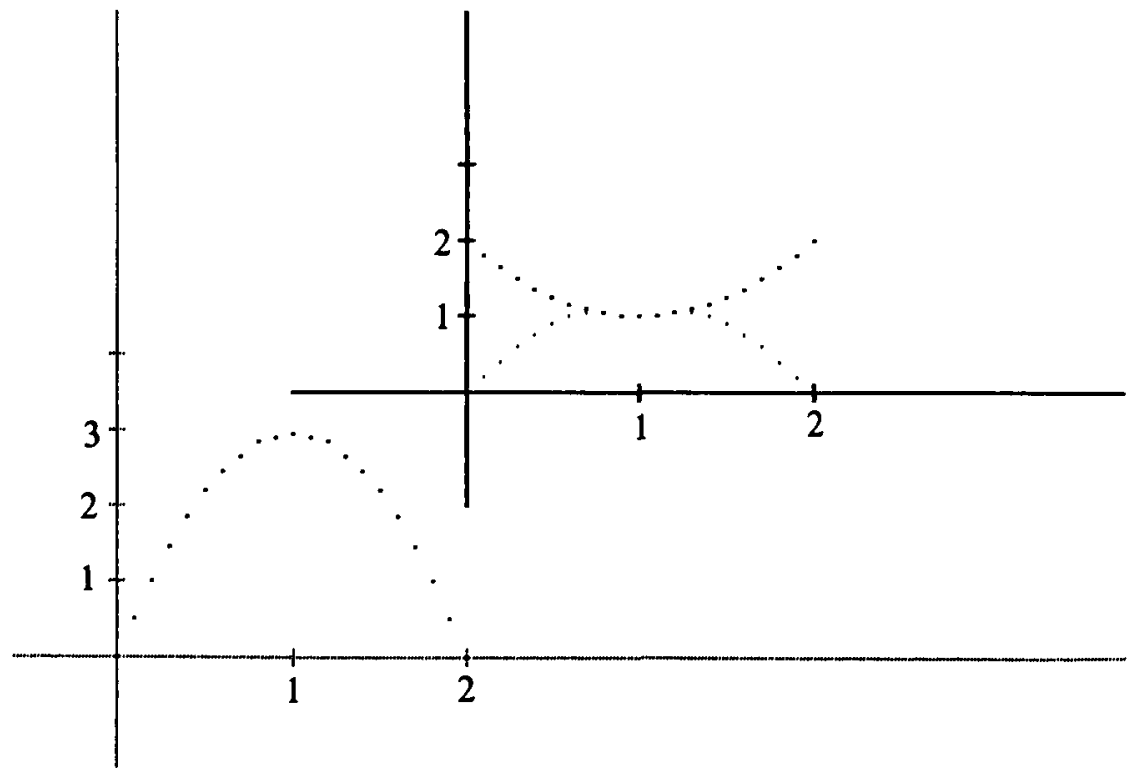

FIgURE 1 
solution of the above obstacle problem (right) with that of the same equation without the obstacle (left).

\section{Acknowledgement}

Wc wish to thank the referee for useful suggestions.

\section{References}

[1] S. Agmon, A. Douglis and L. Nirenberg, "Estimates near the boundary for solutions of elliptic partial differential equations satisfying general boundary conditions", I, Comm. Pure Appl. Math. 12 (1959) 623-727.

[2] H. Amann, "Fixed point equations and nonlinear eigenvalue problems in ordered Banach spaces", SIAM Rev. 18 (1976) 620-709.

[3] L Boccardo and D. Giachetti, "Strongly nonlinear unilateral problems", Appl. Math. Optimaization 9 (1983) 291-301.

[4] H. Brezis and F. E. Browder, "Strongly nonlinear elliptic boundary value problems", Annali Scuola Norm. Sup. Pisa (1978) 587-603.

[5] L. A. Caffarelli, "The regularity of free boundaries in higher dimension, Acta Math.", 139 (1977) 155-184.

[6] A. Friedman, Variational principles and free-boundary problems (John Wiley and Sons, New York, 1982).

[7] D. Gilbarg and N. S. Trudinger, Elliptic partial differential equations of second order (Springer-Verlag, New York, 1977).

[8] R. Glowinski, Lectures on numerical methods for non-linear variational problems (TATA Institutie, Bombay, 1980).

[9] H. B. Keller, "Some positone problems suggested by nonlinear heat generation", in Bifurcation theory and nonlinear eigenvalue problems, J. B. Keller and S. Antman Eds., (Benjamin, New York, 1969).

[10] D. Kinderlehrer and L. Nirenberg, "Regularity in free boundary problems", Ann. Scu. Norm. Sup. Pisa 4(4) (1977) 373-391.

[11] A. Leung, "Monotone schemes for semilinear elliptic systems related to ecology", Math. in Appl. Sci. 4 (1982) 272-285.

[12] A. W. Leung and D. A. Murio, "Accelerated monotone scheme for finite difference equations concerning steady-state prey-predator interactions", J. of Comput. and Appl. Math. 16 (1986) 333-341. 